\title{
Technical Analysis of Reliability Design of Electronic Communication Equipment
}

\author{
Wang JinYu \\ Shanghai Civil Aviation College, China,200232
}

Keywords: Electronic communication; reliability; design technology

\begin{abstract}
The reliability of electronic communication equipment will be improved, and it will be upgraded from the source of product design. On the one hand, the product quality will be improved, on the other hand, the development cost will be reduced and the product market competitiveness will be improved. The article focuses on the reliability technology design of electronic equipment, and focuses on the design techniques, EMC design techniques and software reliability design techniques for ensuring reliability indicators.
\end{abstract}

Reliability design is a key part of the reliability assurance system of modern electronic equipment. The basic principles and implementation methods of reliability design of electronic equipment are expounded, including the reliability selection of components, the reliability design of electronic circuits and the reliability design of printed circuit boards.

\section{The basic tasks and significance of reliability design}

\subsection{Basic tasks of reliability design}

In general, the basic task of the reliability design of electronic communication equipment is: based on the current level of components, from the overall design of the equipment, thermal design, environmental design, stability design, maintenance design, etc. By taking different measures, the weight, performance, weight and other factors are balanced to achieve equipment reliability indicators[1].

\subsection{The significance of reliability design}

In short, the reliability design of electronic communication equipment is expressed in the following aspects: reliability exists throughout the life cycle of electronic communication equipment, whether for the design of equipment, or the installation, use and maintenance of equipment. Can not be separated from reliability. However, an important premise is to pay attention to the reliability design, that is, the reliability index of the equipment should be strictly reflected in the design process, in order to provide favorable conditions for the inherent reliability of the equipment. If the reliability design of electronic communication equipment is not taken into account during the application process, the quality of the electronic communication equipment cannot be ensured. According to relevant statistics, the gradual development due to unreasonable design has become an important factor affecting the reliability of equipment. With the rapid development of science and technology, electronic communication equipment is gradually developing towards intelligent, miniaturized and multi-functional. As the function continues to evolve, the reliability requirements for components continue to increase. Therefore, it is required to scientifically select components and implement technologies such as cooling and derating to effectively reduce the efficiency of components, so as to ensure the reliability of electronic communication equipment. Pay attention to the reliability measures in the design process of products, and in the later stage. The measures taken at the stage have an important role and significance, which not only can effectively reduce energy consumption, but also have significant effects. 


\section{Main factors affecting the reliability of electronic communication equipment}

\subsection{Influence of mechanical conditions on electronic communication equipment}

Specifically, the mechanical condition refers to the mechanical action of the impact and vibration of the different vehicles during the use of the electronic communication device. The impact on the equipment is usually manifested by component damage failure, structural deformation and metal parts damage[2].The factors affecting the reliability of electronic communication equipment are as follows.

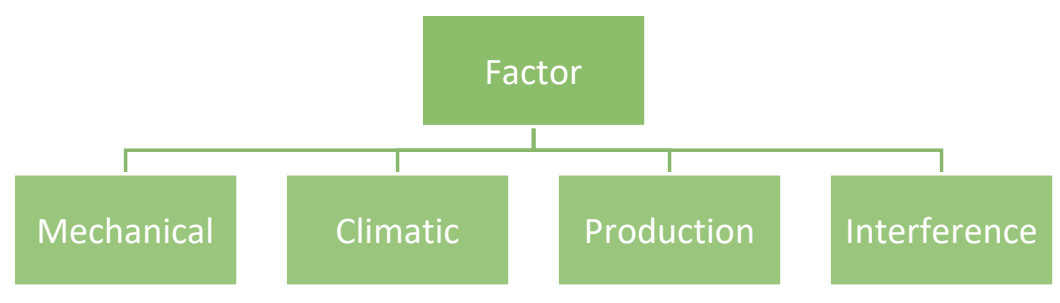

Figure 1: Factors affecting the reliability of electronic communication equipment

\subsection{Impact of climatic conditions on electronic communication equipment}

In general, climatic conditions cover factors such as air pressure, temperature, sunshine, and air pollution. The impact on electronic communication equipment usually results in reduced electrical performance, damage to structures, inflexibility of devices, and, in serious cases, electronic communication. The device is not working properly.

\subsection{Influence of production conditions on electronic communication equipment}

Often, electronic equipment needs to be applied to production activities after successful development. The production conditions include the equipment status, production capacity, production technology, production management level, etc. of the production plant. If the production equipment is to be put into production smoothly, it is necessary to meet all the requirements of the production conditions, so that it can be better invested. To production activities.

\subsection{Influence of electromagnetic interference on electronic communication equipment}

It is well known that in the course of working, electronic communication equipment is inevitably affected by various electromagnetic waves, which may interfere with the inside or outside of the equipment. Electromagnetic interference will cause the noise of electronic communication equipment to increase, and it is impossible to ensure that the equipment works stably or even safely[3].

\section{Countermeasures to improve the reliability design of electronic communication equipment}

Since the reliability design of electronic communication equipment plays an important role in ensuring the quality of electronic communication equipment, it is particularly important to improve the reliability design of electronic communication equipment. How to effectively improve the reliability design of electronic communication equipment, you can start from the following aspects:

\subsection{Scientific selection of electronic components}

First, we must fully consider the circuit performance and the actual requirements of the working environment, and at the same time require that the selected components should meet the equipment working requirements in terms of quality and technical performance, and ensure that there is a 
certain margin;

Second, we should try to select those components that have been proven to be reliable and reliable, and do not use components that are not up to standard and eliminated[4].

Third, effectively compress the variety specifications of components and minimize the manufacturer, so as to improve the reuse rate;

Fourth, under normal circumstances, electronic components that meet relevant requirements should be applied to electronic communication devices after being screened for reliability;

Finally, for the same type of components of different specifications, we must carefully analyze the differences between these components, and choose the preferred. At the same time, it is necessary to consciously collect data on the performance and reliability of components during use, which will provide a strong reference for future selection.

\subsection{Electromagnetic protection of electronic communication equipment}

Under normal circumstances, shielding refers to the form of a plate or a box made of conductive or magnetically permeable materials, which organically controls the electromagnetic field within a specific space, so that the electromagnetic field can be transmitted from one side of the shield to the other. Severe attenuation, effectively controlling the diffusion of electromagnetic fields. Shielding can be divided into electrical shielding, electromagnetic shielding, and magnetic shielding according to the difference in the suppression function. Specifically, electrical shielding, also known as electric field or electrostatic shielding, is mainly used to suppress parasitic capacitance coupling, thereby effectively isolating static electricity and preventing electric field interference. The most common method of electrical shielding is to insert a well-grounded metal plate between the sensing source and the sensor, so that the parasitic capacitance of the sensing source can be shorted to the ground for shielding; electromagnetic shielding is mainly to prevent high frequency. Interference caused by electromagnetic fields; the main purpose of magnetic shielding is to prevent magnetic induction and to isolate magnetic field interference[5].

\subsection{Heat dissipation measures for electronic equipment}

The natural heat dissipation of the casing refers to the mechanical structure that accepts the heat inside the equipment and spreads it to the nearby environment. Generally speaking, the heat dissipation of the chassis should consider the following contents: First, the materials with better thermal conductivity should be selected as much as possible for the casing, so as to effectively enhance the heat conduction of the inner and outer surfaces of the chassis; second, in the casing The inner and outer surfaces are coated with a black lacquer to enhance the heat radiation capability of the casing. Third, a scientific and reasonable air hole is placed on the casing to improve the convective heat transfer capacity of the airflow. .

\subsection{Maintainability design of electronic communication equipment}

Under normal circumstances, electronic communication equipment is a product that can be repaired. At the same time, high requirements are also placed on this type of product. It is necessary to have as few failures as possible, and to be repaired in time when a failure occurs. In other words, in order to effectively improve the utilization rate of electronic communication equipment, it is necessary to strictly implement electronic communication equipment as little as possible, and even if a failure occurs, it should be repaired in the first time. For maintenance design, we must focus on: easy to disassemble when repairing; ensure the reliability of maintenance tools; have good interchangeability.

\section{Conclusion}

Reliability is the focus of design, and reliability management reflects the proficiency in the manufacturing process. Therefore, it is necessary to increase the collection of data in the production process, thereby gradually improving the production quality and improving the stability of equipment use. In summary, regarding the reliability design of electronic equipment, we must focus 
on implementation and details. We must have achieved certain results in terms of reliability. However, we still need to continuously summarize our experience. We need a certain amount of practice, time, experience, and a large amount of data accumulation to better ensure the reliability design of electronic equipment. .

\section{References}

[1] Li Fu, Xiu Wei Fu, Jing Li. Design of Platform for Digital Electronic Technology Based on EP3C5E[J]. Advanced Materials Research,2014,3181(926).

[2] Pai Peng, Yong Jiang Zhong, Yu Han Zhang. The Design and Research of Electronic Schoolbag Learning Environment Take "Science of Eyes" of the Science of Primary School for Exam[J]. Applied Mechanics and Materials,2014,3512(651).

[3] Josep M. Oliva, Douglas J. Klein, Paul von Ragué Schleyer, Luis Serrano-Andrés. Design of carborane molecular architectures with electronic structure computations: From endohedral and polyradical systems to multidimensional networks[J]. Pure and Applied Chemistry,2013,81(4).

[4] FUKUE Takashi, KOIZUMI Katsuhiro, HATAKEYAMA Tomoyuki, ISHIZUKA Masaru, NAKAGAWA Shinji. D314 STUDY ON P-Q CURVES OF COOLING FANS FOR THERMAL DESIGN OF ELECTRONIC EQUIPMENT: EFFECTS OF OPENING POSITION LOCATED IN FRONT OF A FAN(Components-3)[J]. The Proceedings of the International Conference on Power Engineering (ICOPE),2009,2009.3(0).

[5] Yazyev Oleg V. A Guide to the Design of Electronic Properties of Graphene Nanoribbons. [J]. Accounts of Chemical Research,2013. 